\title{
Estimation of index of power law rigidity spectrum of cosmic rays using effective rigidity of multidirectional muon detector
}

\author{
G. Kalugin* and K. Kabin
}

\begin{abstract}
We develop a new approach for calculating index of power law for variations in the differential rigidity spectrum of cosmic rays registered by Nagoya multidirectional muon detector. Our approach is based on effective rigidity which we define for the entire instrument, not just for its individual channels. Our definition of effective rigidity can be straightforwardly extended to other multichannel instruments and networks of instruments. As examples, we calculate the power law index under quiet conditions and during 12 Forbush decrease events. We also discuss sensitivity of the Nagoya muon detector to different values of the power law index and different rigidities.
\end{abstract}

Keywords: Cosmic rays; Multidirectional muon detector; Coupling coefficients; Effective rigidity

\section{Findings}

\section{Introduction}

The ability of cosmic ray (CR) particles to penetrate magnetic fields and reach the top of the Earth's atmosphere is controlled by their rigidity $R$ which is defined as the particle's momentum multiplied by the speed of light per unit charge. The rigidity is usually measured in gigavolts (GV). As CRs propagate through the solar system, they interact with the interplanetary magnetic field (IMF) and their fluxes are modulated by its intensity and direction. Fluxes of CRs with different rigidities change differently since lower rigidity particles are more affected by the changes in the magnetic field. Thus, variations $\delta D(R) / D(R)$ in the differential rigidity spectrum $D(R)$ of CRs are related to changes in power spectral density of IMF turbulence (Wawrzynczak and Alania 2009) and thereby related to fundamental properties of the solar wind and IMF, which are important for space weather and astrophysical applications (Dorman 2006). Variations $\delta D(R) / D(R)$ are often well approximated by a power law (Dorman 1974; Fujimoto et al. 1984).

Common ground based detectors used to monitor CR fluxes are neutron monitors and muon detectors

*Correspondence: German.Kalugin@gmail.com

Department of Physics, Royal Military College of Canada, PO Box 17000 SF, Kingston, Ontario, Canada measuring, respectively, secondary CR neutrons and muons produced in the atmosphere. These instruments are most sensitive to $C R$ particles in different energy ranges, typically $\sim 10 \mathrm{GeV}$ for a neutron monitor and $\sim 50$ $\mathrm{GeV}$ for a muon detector (Leerungnavarat et al. 2003), and therefore they represent complementary tools for a study of CRs properties. In this paper, we consider Nagoya multidirectional muon detector located at $35^{\circ} 09^{\prime} \mathrm{N}, 136^{\circ}$ $58^{\prime} \mathrm{E}$ and $77 \mathrm{~m}$ above the sea level (STEL site 2015). The Nagoya muon detector has 17 channels listed in Table 1. Symbol 'V' refers to the vertical component, 'N30' denotes the direction pointing toward the north with zenith angle $30^{\circ}$ and so on. Table 1 shows the average counting rate $N_{0}$ for each channel, the statistical mean error of measurements $\sigma$, and the cutoff and median rigidities $R_{\mathrm{c}}$ and $R_{\mathrm{m}}$ (Okazaki et al. 2008). The last column of the table is explained in the section "Additional remarks".

In this paper, we assume a power law for variations $\delta D(R) / D(R)$ in the differential rigidity spectrum of CRs registered by the Nagoya muon detector and develop a new approach for finding its power law index based on the effective rigidity of the detector. We present the calculations using this method for cases corresponding to both quiet and disturbed conditions. We also discuss the concept of the effective rigidity of the detector and its implications on the sensitivity of the instrument.

\section{Springer}

(c) 2015 Kalugin and Kabin. Open Access This article is distributed under the terms of the Creative Commons Attribution 4.0 International License (http://creativecommons.org/licenses/by/4.0/), which permits unrestricted use, distribution, and reproduction in any medium, provided you give appropriate credit to the original author(s) and the source, provide a link to the Creative Commons license, and indicate if changes were made. 
Table 1 Characteristics of channels of the Nagoya muon detector

\begin{tabular}{llllll}
\hline Channels & $N_{0}, 10^{6}$ counts/h & $\sigma, \%$ & $R_{c}, G V$ & $R_{m}, G V$ & $\gamma_{m}$ \\
\hline V & 2.76 & 0.06 & 11.5 & 59.4 & - \\
N30 & 1.25 & 0.09 & 12.9 & 64.6 & -1.13 \\
E30 & 1.20 & 0.09 & 16.2 & 66.7 & -0.97 \\
S30 & 1.23 & 0.10 & 11.3 & 62.6 & - \\
W30 & 1.26 & 0.09 & 9.4 & 61.8 & - \\
NE39 & 0.58 & 0.14 & 17.9 & 72.0 & -1.03 \\
SE39 & 0.58 & 0.14 & 15.0 & 69.3 & -1.14 \\
SW39 & 0.60 & 0.13 & 9.2 & 65.6 & - \\
NW39 & 0.62 & 0.13 & 11.0 & 66.6 & -1.68 \\
N49 & 0.61 & 0.13 & 12.9 & 83.0 & -1.24 \\
E49 & 0.58 & 0.14 & 21.0 & 88.3 & -1.10 \\
S49 & 0.60 & 0.13 & 10.9 & 80.5 & -1.39 \\
W49 & 0.62 & 0.13 & 9.3 & 79.3 & -1.55 \\
N64 & 0.18 & 0.27 & 11.0 & 105.0 & -1.20 \\
E64 & 0.17 & 0.28 & 25.1 & 113.7 & -1.10 \\
S64 & 0.18 & 0.27 & 10.8 & 103.7 & -1.25 \\
W64 & 0.18 & 0.27 & 8.7 & 103.0 & -1.29 \\
\hline & & & & & \\
\hline
\end{tabular}

\section{Coupling coefficients}

The differential rigidity spectrum of CRs has been studied extensively and is well described by power law $D(R) \sim$ $R^{-\alpha}$ where, for example, for protons $\alpha \approx 2.82$ for a wide rigidity range from 30 to $1000 \mathrm{GV}$ (e.g., Adriani et al. 2011). Variations in this spectrum can be caused by changes in solar activity, coronal mass ejections, and other sources causing changes in the IMF (Dorman 1974). Often, such disturbances produce a temporary decline in the CR intensity called Forbush decrease (FD). Heliospheric and magnetospheric modulations of the intensity of CRs depend on the rigidity of CR particles with low-rigidity particles being affected more than the highrigidity ones. These variations in the differential rigidity spectrum $\delta D(R) / D(R)$ are also commonly assumed to be a power law with exponent $\gamma$ (Dorman 1974; Fujimoto et al. 1984)

$$
\frac{\delta D(R)}{D(R)}= \begin{cases}(R / 10)^{\gamma}, & \text { for } R<R_{\mathrm{u}} \\ 0, & \text { for } R>R_{\mathrm{u}}\end{cases}
$$

where $R$ is in GV and $R_{\mathrm{u}}$ is the upper threshold rigidity beyond which the modulation effect vanishes. Following Dorman (1974); Cane (2000); Wawrzynczak et al. (2006); Kravtsova and Sdobnov (2013) as well as others, we assume that $\gamma<0$ for both quiet and disturbed conditions, including FDs. The power law index, $\gamma$, is very important in the CR studies because it is related to the characteristics of the process of scattering cosmic rays by magnetic irregularities and especially by turbulence (Wawrzynczak et al. 2006). Observations of CR intensity at the ground level are connected with the variations of the rigidity spectrum through the so-called coupling coefficients which depend on the characteristics of the detector as well as $\gamma$ and $R_{\mathrm{u}}$ which may be different for different events. Specifically, the isotropic part $v_{0, i}(t)$ of the flux observed with the $i$-th channel of the Nagoya muon detector is proportional to the coupling coefficient $c_{0, i}^{0}$ (Fujimoto et al. 1984)

$$
\begin{aligned}
& v_{0, i}(t)=a_{0}(t) c_{0, i}^{0}, \\
& c_{0, i}^{0}=\int_{R_{\mathrm{c}, i}}^{\infty} \frac{\delta D(R)}{D(R)} W_{i}\left(R, R_{\mathrm{m}, i}\right) d R,
\end{aligned}
$$

where $a_{0}(t)$ is the amplitude of variation in space and $R_{\mathrm{c}, i}$, $R_{\mathrm{m}, i}$ and $W_{i}\left(R, R_{\mathrm{m}, i}\right)$ are the cutoff rigidity, median rigidity, and coupling function of the $i$-th viewing channel, respectively.

The values of $c_{0, i}^{0}$ are computed by Fujimoto et al. (1984) under assumption (1) and are available as a table for $\gamma=-2,-1.5,-1,-0.5,0$ and 0.5 and for $R_{u}=30 \mathrm{GV}$, $50 \mathrm{GV}, 100 \mathrm{GV}, 200 \mathrm{GV}, 500 \mathrm{GV}$ and $1000 \mathrm{GV}$. For convenience of our analysis, we interpolate the $\gamma$-dependence of these data points with hyperbolas. We find that the standard deviation of this approximation is less than 0.01 .

We also note that in our calculations, we analyze only the isotropic part of CR intensity. However, the Nagoya muon detector measurements have also been used for studies of anisotropy of CRs, for example, by Kozai et al. (2014) who derived the yearly mean values for the threedimensional anisotropy and the modulation parameters and found it to be consistent with those derived from the Global Muon Detector Network data.

\section{Effective rigidity}

The effective rigidity of a channel is defined as the rigidity level at which the variations in CR fluxes are the same as the variations integrated over the whole energy range (Plainaki et al. 2007). This effective rigidity can be computed as

$$
\nu_{0, i}(t)=a_{0}(t)\left(R_{e f f, i} / 10\right)^{\gamma},
$$

where $R_{e f f, i}$ is in GV. Thus, $R_{e f f, i}$ is related to the coupling functions and coupling coefficients as follows

$$
R_{e f f, i}=10\left(\int_{R_{\mathrm{c}, i}}^{R_{\mathrm{u}}}(R / 10)^{\gamma} W_{i}\left(R, R_{\mathrm{m}, i}\right) d R\right)^{1 / \gamma}=10\left(c_{0, i}^{0}(\gamma)\right)^{1 / \gamma} .
$$

Therefore, the effective rigidity of a muon detector channel depends on both the characteristics of the detector and the considered event (through values of $\gamma$ and $R_{u}$ ).

We note that the effective rigidity defined above is a completely different concept from the effective cutoff rigidity used, for example, by Cooke et al. (1991) to describe the magnetospheric transmissivity (Kudela and Usoskin 2004) and penumbral structure of the CRs. This 
effective cutoff rigidity typically has a much lower value than the effective rigidity described here.

In addition to the effective rigidity of individual channels, it is possible to introduce the effective rigidity of an entire detector as an average of those of all the channels. It is natural to use standard deviations of the channels (from Table 1) in this definition:

$\left\langle R_{e f f}\right\rangle=\left(\frac{\sum_{i=1}^{17} \sigma_{i}^{-2} R_{e f f, i}^{\gamma}}{\sum_{i=1}^{17} \sigma_{i}^{-2}}\right)^{1 / \gamma}=10\left(\frac{\sum_{i=1}^{17} \sigma_{i}^{-2} c_{0, i}^{0}(\gamma)}{\sum_{i=1}^{17} \sigma_{i}^{-2}}\right)^{1 / \gamma}$.

This, however, is not the only possible choice for the definition of the effective rigidity of a detector; another definition with different weights is discussed later.

The dependence (3) is shown in Fig. 1 for $R_{\mathrm{u}}=100,200$, 500 and $1000 \mathrm{GV}$ by squares, triangles, crosses, and circles, respectively. It is a monotonically increasing function of $\gamma$ which has a removable singularity at $\gamma=0$. For example, for $R_{\mathrm{u}}=1000 \mathrm{GV},\left\langle R_{e f f}\right\rangle(\gamma=0)=115.49 \mathrm{GV}$. We find that $\left\langle R_{e f f}\right\rangle$ dependence on $\gamma$ is well fitted by hyperbola

$$
\left\langle R_{e f f}\right\rangle=\frac{p}{q-\gamma}+r \quad(-2 \leq \gamma \leq 0),
$$

where coefficients $p, q$, and $r$ depend on $R_{\mathrm{u}}$ (between 100 and $1000 \mathrm{GV}$ ) as follows

$$
\begin{aligned}
& p=31.95-\frac{3339.01}{R_{u}+83.53}, q=0.285+\frac{28.89}{R_{u}+21.72}, \\
& r=24.95+\frac{19.45}{R_{u}-168.58} .
\end{aligned}
$$

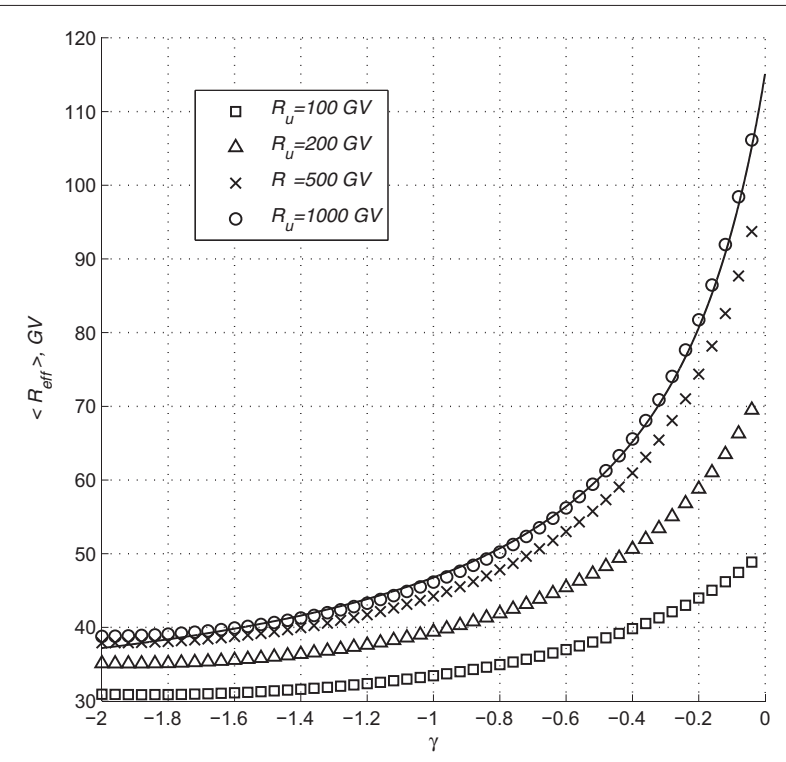

Fig. 1 Effective rigidity of the Nagoya muon detector vs. $\gamma$ computed by formula (3) for $R_{\mathrm{u}}=100 \mathrm{GV}$ (squares), $200 \mathrm{GV}$ (triangles), $500 \mathrm{GV}$ (crosses), and $1000 \mathrm{GV}$ (circles) and by formula (4) for $R_{\mathrm{u}}=1000 \mathrm{GV}$ (solid line)
In these formulas $\left\langle R_{e f f}\right\rangle, R_{\mathrm{u}}, p$, and $r$ are in gigavolts; $q$ is the dimensionless coefficient. The error of this approximation is increasing with $R_{\mathrm{u}}$ but does not exceed $2 \%$. As an example, fitting for $R_{\mathrm{u}}=1000 \mathrm{GV}$ is shown in Fig. 1 by solid line.

\section{Calculating the power law index using the effective rigidity} The effective rigidity can be used to compute the power law exponent $\gamma$. We start by defining the isotropic part of the intensity recorded by a muon detector averaged over its channels as (Dorman 1974, p. 40)

$$
\left\langle v_{0}\right\rangle=\frac{\sum_{i=1}^{17} v_{0, i} \sigma_{i}^{-2}}{\sum_{i=1}^{17} \sigma_{i}^{-2}} .
$$

Note that definitions (3) and (5) are consistent in the sense that they give relation

$$
\left\langle v_{0}\right\rangle=a_{0}(t)\left(\left\langle R_{e f f}\right\rangle / 10\right)^{\gamma},
$$

which is similar to (2).

For practical considerations, it is convenient to normalize this quantity by the intensity measured by the vertical channel, i.e., introduce ratio (Kalugin and Kabin 2015)

$$
\langle k\rangle=\left\langle\nu_{0}\right\rangle / \nu_{0,1} .
$$

By definitions (2) and (3), this ratio is related to the average effective rigidity as

$$
\langle k\rangle=\left(\left\langle R_{e f f}\right\rangle / R_{e f f, 1}\right)^{\gamma} .
$$

Therefore, if $\langle k\rangle$ is computed from data, the corresponding value of $\gamma$ is determined from Eq. 7. To avoid temperature and noise effects, we can use 24-hour moving average intensity recorded in the $i$-th directional channel (e.g. Okazaki et al. 2008).

Dependence of $\left(\left\langle R_{e f f}\right\rangle / R_{e f f, 1}\right)^{\gamma}$ for $R_{u}=1000 \mathrm{GV}$ is shown in Fig. 2 by a dashed line. It is a monotonically increasing function of $\gamma$, and therefore, Eq. (7) has a single solution.

We first demonstrate computing $\gamma$ under quiet geomagnetic conditions using day of the year (DOY) $223 / 2011$ as an example. For this day, the planetary index $K_{\mathrm{p}}$ (Rostoker 1972) did not exceed $1 \frac{2}{3}$, and for the previous 3 days $K_{\mathrm{p}}$ did not exceed 3 (NGDC site 2015). For the quiet conditions, we define intensity variation $v_{0, i}$ as relative changes in count rates referred to the minimum value of counts during the day. Taking averages over a day, we obtain, using (5), $\left\langle v_{0}\right\rangle=0.025 \%$. Since $v_{0,1}=0.026 \%$ for this day, we get $\langle k\rangle=0.96$ and, finally, $\gamma=-0.3$, which agrees well with the typical values of the perturbation spectrum index under quiet conditions (Kane, 1963). In addition, once spectrum index is known, the amplitude of the variation can be found using Eq. 6. Specifically, from Eq. 3, $\left\langle R_{\text {eff }}\right\rangle=72.45 \mathrm{GV}$ for $\gamma=-0.3$ and, thus, the amplitude averaged over the day is $a_{0}=0.046 \%$. 


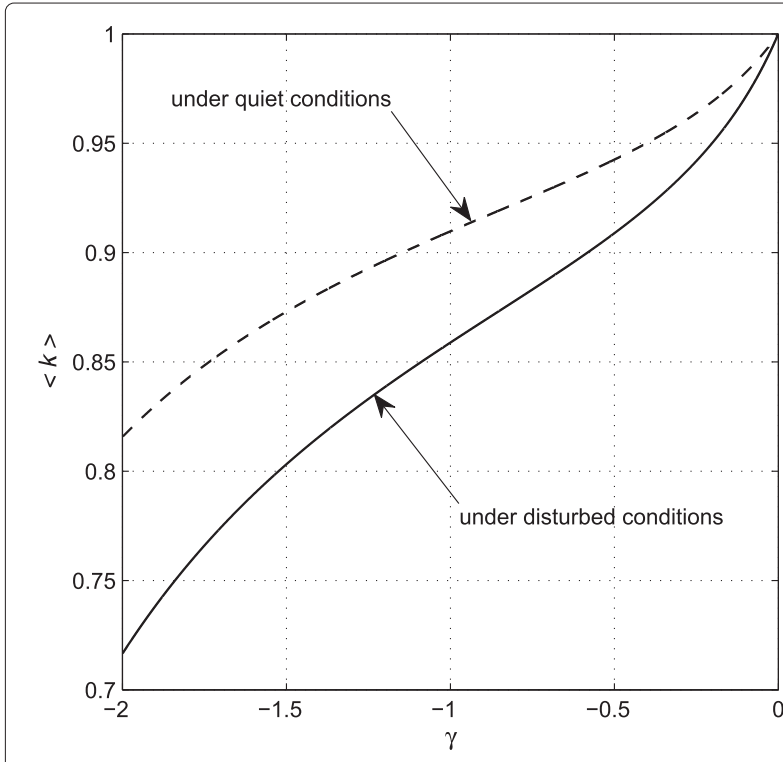

Fig. 2 Normalized averaged intensity of the Nagoya muon detector vs. spectrum index derived under quiet (dash line) and disturbed (solid line) conditions

We can also apply this approach to measurements made under disturbed conditions during FD events. However, since most FDs last only 2-3 days, in this case we analyze data averaged over an hour, rather than over a day. We consider twelve FD events selected by Kalugin and Kabin (2015) and listed in Table 2. The table includes the DOYs, when they occurred, and values of $\langle k\rangle$ computed from the detector data. The lines with $n$ and $\gamma_{\sigma}$ show values of the rigidity spectrum index calculated by Kalugin and Kabin (2015) and using the above method, respectively. One can see a significant difference, often exceeding $35 \%$, between these values. In this calculation, we used $R_{\mathrm{u}}=1000 \mathrm{GV}$. If a lower value of $R_{\mathrm{u}}$ is used, the differences increase even further.

It is, however, possible to modify the averaging procedure in formula (3) by replacing weights $\sigma_{i}^{-2}$ with $k_{i}$ which is the ratio of FD amplitude measured by $i$-th channel to that measured by the vertical channel, see (Kalugin and
Kabin 2015). Choosing $k_{i}$ values as weights is similar to the approach used by Plainaki et al. (2007) for study of ground level enhancement events. The values of the spectral index calculated using this approach are shown in Table 2 as $\gamma_{k}$. The difference between $n$ and $\gamma_{\mathrm{k}}$ is smaller than before and for half of the events, particularly, for events No. 2, $5,8,9,10$, and 11 , it does not exceed $7 \%$. Thus, for disturbed conditions, this may be a better choice than (3) for defining the average effective rigidity of a detector.

We also found that the curves obtained with using weights $k_{i}$ for different events are very close to each other. Therefore, we computed the mean of these curves shown in Fig. 2 by solid line. The values of the spectral index obtained using this average curve for all events are shown in the last line of Table 2; the difference between $\gamma_{\mathrm{k}}$ and $\tilde{\gamma}_{\mathrm{k}}$ does not exceed $5 \%$.

Finally, we note that both curves in Fig. 2 are quite similar, and, in particular, they have an inflection point at $\gamma=-0.85$. They can be fitted well by cubic parabolas:

$$
\begin{gathered}
\langle k\rangle_{\text {quiet }}=0.028 \gamma^{3}+0.08 \gamma^{2}+0.14 \gamma+0.99 \\
\langle k\rangle_{\mathrm{FD}}=0.044 \gamma^{3}+0.13 \gamma^{2}+0.22 \gamma+0.99
\end{gathered}
$$

The standard deviation for these fits to Eq. 7 is less than 0.002. Approximation (8) worked well for all the events listed in Table 2 and can be expected to be adequate for other FD events as well.

\section{Additional remarks}

In this section, we discuss some additional properties of the effective rigidity which, while are not directly connected with the problem of computing the power law index, may be interesting for CR studies using mutidirectional instruments.

First, we note that the value of the median rigidity of the vertical channel, which is the most statistically significant one, is $59.4 \mathrm{GV}$. The effective rigidity $\left\langle R_{\text {eff }}\right\rangle$ reaches this value at $\gamma=-0.5$. Thus, the muon detector is expected to be most sensitive to $C R$ variations with spectrum index close to -0.5 or, equivalently, when the normalized averaged intensity is close to 0.94. Since the derivative of

Table 2 Rigidity spectrum index for 12 Forbush decreases

\begin{tabular}{lllllllllllll}
\hline No. & 1 & 2 & 3 & 4 & 5 & 6 & 7 & 8 & 9 & 10 & 11 & 12 \\
Year & 2003 & 1998 & 2005 & 2001 & 2004 & 2000 & 2000 & 2001 & 2005 & 1998 & 2001 & 2001 \\
DOY & 302 & 268 & 135 & 118 & 22 & 160 & 195 & 86 & 254 & 312 & 310 & 229 \\
\hline$\langle k\rangle$ & 0.88 & 0.86 & 0.89 & 0.88 & 0.85 & 0.87 & 0.86 & 0.87 & 0.85 & 0.84 & 0.83 & 0.81 \\
$n$ & -1.07 & -1.04 & -0.89 & -0.91 & -1.11 & -1.09 & -1.11 & -0.98 & -1.13 & -1.28 & -1.33 & -1.23 \\
$\gamma_{\sigma}$ & -1.45 & -1.66 & -1.36 & -1.45 & -1.71 & -1.53 & -1.65 & -1.58 & -1.74 & -1.82 & -1.89 & -2.0 \\
$\gamma_{k}$ & -0.80 & -0.99 & -0.70 & -0.77 & -1.07 & -0.88 & -1.00 & -0.91 & -1.10 & -1.23 & -1.33 & -1.49 \\
$\tilde{\gamma}_{k}$ & -0.81 & -1.01 & -0.73 & -0.81 & -1.06 & -0.89 & -0.99 & -0.93 & -1.09 & -1.18 & -1.27 & -1.46 \\
\hline
\end{tabular}


$\left\langle R_{e f f}\right\rangle$ increases with $\gamma$, the Nagoya muon detector is less sensitive to $C R$ variations with small spectrum index.

Second, the ratio of the effective rigidity of the detector to that of the vertical channel, shown in Fig. 3, has a shallow minimum at $\gamma=-1.39\left(\left\langle R_{\text {eff }}\right\rangle=41.36 \mathrm{GV}\right.$ at this $\gamma$ ). Similarly, the effective rigidities of most individual channels normalized by the vertical one also exhibit a minimum. The corresponding values of $\gamma$ are listed in the last column in Table 1. Interestingly, the average of these values is -1.24 , which is very close to -1.27 , the average value of the power law exponent for moderate FDs found by Kalugin and Kabin (2015). It is not clear if this is just a coincidence.

The non-monotone behavior of the normalized effective rigidities is explained by the interplay between the coupling functions and cutoff rigidities of different channels. The only channels for which $R_{\text {eff, }, i} / R_{\text {eff }, 1}$ monotonously increases with $\gamma$ are S30, W30, and SW39. These three channels are closest to the vertical channel in terms of measured fluxes and the shape of the coupling functions and are characterized by low cutoff rigidities. The fact that the behavior of channels S30, W30, and SW39 is different from all the others suggests that in some future studies of CRs using the Nagoya muon detector, these channels may be combined with the vertical channel, while the other are treated separately. This argument can be used, for example, to define identical channels to increase the statistical significance of certain measurements (Alaniya et al. 1975). Similar considerations for a detector with many solid angle bins may be used for regrouping data recorded in fine solid angle bins into data in coarse bins (Subramanian et al. 2009).

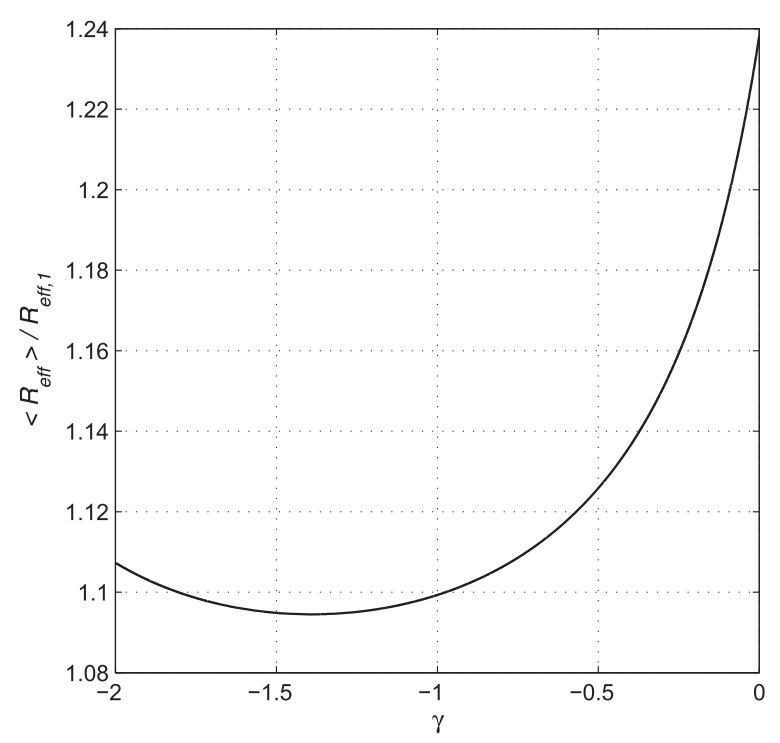

Fig. 3 Normalized effective rigidity of the Nagoya muon detector vs. $\gamma$ computed for $R_{\mathrm{u}}=1000 \mathrm{GV}$
Finally, we note that Eqs. 7 and 3 describe dependence of $\left\langle R_{\text {eff }}\right\rangle$ on $\langle k\rangle$ in a parametric form where $\gamma$ plays a role of parameter. Figure 4 shows this dependence. Not surprisingly, as $\langle k\rangle$ approaches 1 , the effective rigidity increases, since higher rigidity particles are less affected by magnetic field and therefore make a larger contribution to the intensity of particles registered by detector.

\section{Conclusions}

In this paper, we discussed possible definitions of the average effective rigidity for the Nagoya muon detector, which extend the corresponding concept of effective rigidities for individual channels. Connections between the effective rigidity and the differential rigidity spectrum make it a useful data analysis tool. We assumed a power law for the spectrum and develop a new approach for finding the exponent of this power law and estimating the isotropic part of variations in CR intensity using data from the muon detector. We find that this exponent can be easily and effectively calculated using effective rigidity which depends on the CR fluxes measured by the instrument as well as the applicable coupling coefficients. As an illustration of this method, we calculated $\gamma$ for quiet and disturbed conditions using the Nagoya multidirectional muon detector. We also suggest the approximate formulas for the effective rigidity which can simplify this calculation even further.

In addition, we show that certain characteristics of directional channels S30, W30, and SW39 of the Nagoya muon detector are close to that of the vertical channel and different from all the others and therefore, in some future studies, these channels may be combined with the vertical

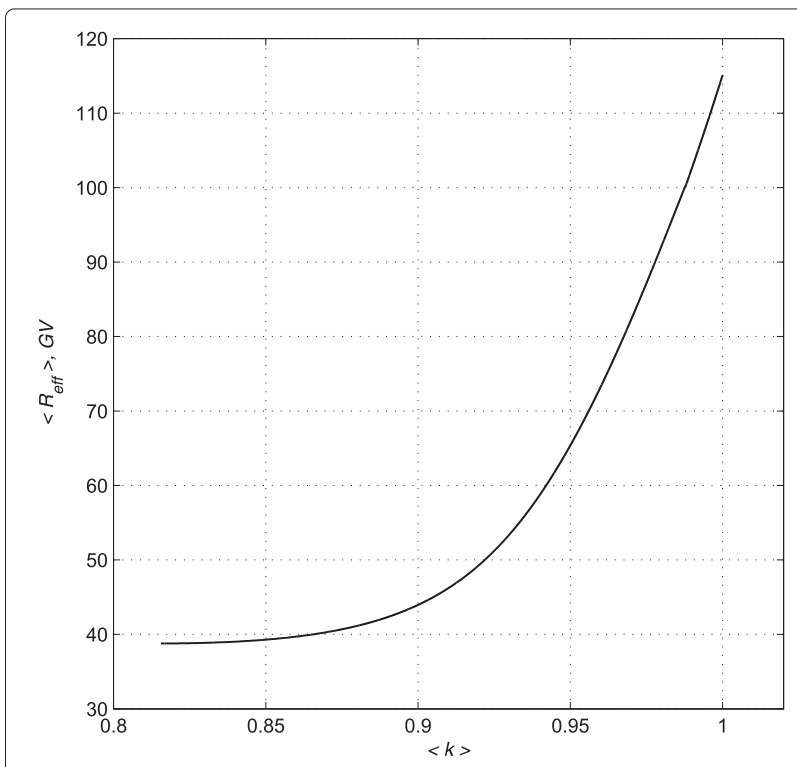

Fig. 4 Effective rigidity against normalized averaged intensity for Nagoya muon detector 
channel, while the others are treated separately. We also showed that the Nagoya muon detector is expected to be the most sensitive to $C R$ spectrum variations characterized by the spectral index close to -0.5 .

Finally, we note that effective rigidity of any multichannel instrument detecting high-energy CRs can be defined similarly to that described in the paper. However, this extension is limited for detectors registering low-energy CRs, such as neutron monitors because the asymptotic directions of low energy CRs strongly depend on the level of the geomagnetic activity and local time of observations (Kudela and Usoskin 2004; Kudela et al. 2008). In particular, change in cutoff rigidity during Forbush decreases significantly depends on the location of neutron monitor (Flückiger et al. 1986; Katsoulakos et al. 2013). Thus, the approach described in this paper can have limits to application to network of such instruments as neutron monitors.

\section{Competing interests}

The authors declare that they have no competing interests.

\section{Authors' contributions}

Both authors contributed to the development of the approach presented in the paper as well as its applications, drafted the manuscript, and approved the final version for submission. Both authors read and approved the final manuscript.

\section{Acknowledgements}

We are thankful to the team of the Solar-Terrestrial Environment Laboratory for providing data from the Nagoya muon detector (STEL site 2015) and to the team of the National Geophysical Data Center (NGDC site 2015) for providing indices of the magnetic activity. We appreciate Dr. K. Munakata for discussions of various issues associated with the Nagoya muon detector. We are also grateful to anonymous reviewers and editor for valuable comments and remarks which significantly improved the paper. The work was supported by the National Sciences and Engineering Research Council of Canada.

Received: 12 May 2015 Accepted: 27 August 2015

Published online: 15 September 2015

\section{References}

Adriani O, Barbarino GC, Bazilevskaya GA, Bellotti R, Boezio M, Bogomolov EA, Bonechi L, Bongi M, Bonvicini V, Borisov S, Bottai S, Bruno A, Cafagna F, Campana D, Carbone R, Carlson P, Casolino M, Castellini G, Consiglio L, De Pascale MP, De Santis C, De Simone N, Di Felice V, Galper AM, Gillard W, Grishantseva L, Jerse G, Karelin AV, Koldashov SV, Krutkov SY, et al. (2011) PAMELA measurements of cosmic-ray proton and helium spectra. Science 332(6025):69-72

Alaniya M, Bochikashvili D, Dzhapiashvili T, Dorman L, Erkomaishvili G, Kvantaliani O, Kobakhidze Z, Tuskiya I, Shatashvili L (1975) System of underground muon telescopes in Tbilisi for studying the cosmic ray variations in the moderate and high energy range. In: Proceedings of 14th ICRC. Munich, Germany. pp 3389-3393

Cane H (2000) Coronal mass ejections and Forbush decreases. Space Sci Rev 93:55-77

Cooke D, Humble J, Shea M, Smart D, Lund N, Rasmussen I, Byrnak B, Goret P, Petrou N (1991) On cosmic-ray cut-off terminology. II Nuovo Cimento C 14(3):213-234

Dorman L (1974) Cosmic Rays: Variations and Space Explorations. Elsevier, New York

Dorman L (2006) Cosmic ray interactions, propagation, and acceleration in space plasmas. In: Astrophysics and Space Science Library. Springer, Netherlands. p 339
Flückiger E, Smart D, Shea M (1986) A procedure for estimating the changes in cosmic ray cutoff rigidities and asymptotic directions at low and middle latitudes during periods of enhanced geomagnetic activity. J Geophys Res 91(A7):7925-7930

Fujimoto K, Inoue A, Murakami K, Nagashima K (1984) Coupling coefficients of cosmic ray daily variations for meson telescopes. Report of Cosmic-Ray Research Laboratory. Nagoya University, Japan. p 9

Kalugin G, Kabin K (2015) An analysis of large Forbush decrease events using phase diagrams of view channels of the Nagoya multidirectional muon telescope. J Atmos Terr Phys 123:124-136

Kane R (1963) Energy spectrum of variations of cosmic-ray daily mean intensity recorded during the I.G.Y. II Nuovo Cimento 27(2):441-467

Katsoulakos G, Millas D, Laoutaris A, Kontiza K, Mavromichalaki H, Eroshenko E, Belov A, Yanke V (2013) Magnetospheric cut-off rigidity changes during the magnetic storms of the years 2011 and 2012. In: Proceedings of 11th HELLAS Conference, Athens, Greece. Available at http://www.helas.gr/ conf/2013/posters_pres.php

Kozai M, Munakata K, Kato C, Kuwabara T, Bieber J, Evenson P, Rockenbach M, Dal Lago A, Schuch N, Tokumaru M, Duldig M, Humble J, Sabbah I, Al Jassar H, Sharma M, Kóta J (2014) The spatial density gradient of galactic cosmic rays and its solar cycle variation observed with the Global Muon Detector Network. Earth Planets Space 66:151

Kravtsova MV, Sdobnov VE (2013) Rigidity spectrum of cosmic ray variations over periods of large Forbush decreases during the 22nd and 23rd solar cycles. Bull Russ Acad Sci Phys 77(5):543-546

Kudela K, Bučík R, Bobík P (2008) On transmissivity of low energy cosmic rays in disturbed magnetosphere. Adv Space Res 42(7):1300-1306

Kudela K, Usoskin I (2004) On magnetospheric transmissivity of cosmic rays. Czech J Phys 54(2):239-254

Leerungnavarat K, Ruffolo D, Bieber J (2003) Loss cone precursors to Forbush decreases and advance warning of space weather effects. Astrophys $J$ 593:587-596

NGDC site (2015) National Geophysical Data Center (NGDC). http://www.ngdc. noaa.gov/stp/geomag/indices.html Accessed 11 Aug 2015.

Okazaki Y, Fushishita A, Narumi T, Kato C, Yasue S, Kuwabara T, Bieber JW (2008) Drift effects and the cosmic ray density gradient in a solar rotation period: first observation with the Global Muon Detector Network (GMDN). Astrophys J 681:693-707

Plainaki C, Belov A, Eroshenko E, Mavromichalaki H, Yanke V (2007) Modeling ground level enhacements: Event of 20 January 2005. J Geophys Res 112(A04102):16

Rostoker G (1972) Geomagnetic indices. Rev Geophys Space Phys 10(4):935-950

STEL site (2015) Nagoya multi-directional muon telescope, Solar-Terrestrial Environment Laboratory. http://www.stelab.nagoya-u.ac.jp/ste-www1/ div3/muon/dbtext22.pdf Accessed 20 Jan 2015

Subramanian P, Antia HM, Dugad SR, Goswami UD, Gupta SK, Hayashi Y, Ito N, Kawakami S, Kojima H, Mohanty PK, Nayak PK, Nonaka T, Oshima A, Sivaprasad K, Tanaka H, Tonwar SC (2009). Forbush decreases and turbulence levels at coronal mass ejection fronts. A \& A 494:1107-1118

Wawrzynczak A, Alania M (2009) On the role of the interplanetary magnetic field turbulence in the formation of the rigidity spectrum of the Forbush decrease of the galactic cosmic rays. In: Proceedings of 21st ECRS. Košice, Slovakia. pp 317-321

Wawrzynczak A, Alania M, Modzelewska R (2006) Features of the rigidity spectrum of galactic cosmic ray intensity during the recurrent Forbush effect. Acta Phys Pol B 37(5):1667-1676 